\title{
A HYBRID INTELLIGENT APPROACH FOR OPTIMAL CONTROL OF SEED CLEANER
}

\author{
Jin Yuan ${ }^{1,2}$, Tao $\mathrm{Yu}^{1}$, Kesheng Wang ${ }^{3}$ \\ ${ }^{I}$ CIMS \& Robot Center of Shanghai University, Shanghai University, China; Email: \\ yuanjin@graduate.shu.edu.cn. ${ }^{2}$ School of Mechanical \& Electronic Engineering, Shandong \\ Agricultural University, China; ${ }^{3}$ Department of Production and Quality Engineering, \\ Norwegian University of Science and Technology,Norway.E-mail:kesheng.wang@ntmu.no
}

\begin{abstract}
This study presents a hybrid intelligent approach to model and optimize a seed cleaning/separating process, which separates seeds varying with different types, varieties and lots in a seed cleaner system (5XZW-1.5). In this controlling scheme, the nonlinear system is modeled by Artificial Neural Network, and optimized by Genetic Algorithms. The uniform design is used to sample datasets. Control results suggest that the intelligent approach is useful for the optimization of such a complex process.
\end{abstract}

Key words: Artificial Neural Networks, Genetic Algorithms, seed cleaner, uniform design.

\section{INTRODUCTION}

In recent years, intelligent control techniques, such as Artificial Neural Networks (ANNs) and Genetic Algorithms (GAs), have been extensively developed in the control of complex processes, to which mathematical approaches are not easily applied [1][2]. ANNs are capable of generating complex mapping between the input and the output space, and thus, arbitrarily complex nonlinear decision boundaries can be formed by these networks. On the other hand, GAs is one of the combinatorial optimization techniques. It searches for an optimal value of a complex objective function by simulating the biological evolutionary process, based on crossover and mutation in genetics [3]. GAs needs only the fitness values to guide its search. However, it is difficult (sometimes impossible) and time consuming to obtain fitness values for some control problems as it is impractical to run a

This project is supported by the National Natural Science Foundation of China under the grant No.50375090.

Please use the following format when citing this chapter:

Yuan, Jin, Yu, Tao, Wang, Kesheng, 2006, in International Federation for Information Processing (IFIP), Volume 207, Knowledge Enterprise: Intelligent Strategies In Product Design, Manufacturing, and Management, eds. K. Wang, Kovacs G., Wozny M., Fang M., (Boston: Springer), pp. 780-785. 
real system for each combination of control parameters. ANNs as a fitness estimator would be an alternative. A well trained ANNs would be used as an objective function for the GAs optimization process and generate the output value based on the input value.

This paper proposes a hybrid intelligent control system integrating ANNs and GAs, which is applied to the optimal control of the gravity seed cleaner. Section 2 describes the optimal control problem. The methods of control system, ANNs modeling, sampling strategy and design of GAs, are presented in section 3. Finally, the results obtained show the usefulness of the hybrid intelligent system.

\section{OPTIMAL CONTROL PROBLEM}

Seed cleaner uses a combination of air, gravity, and screens to separate seed based on different motion principle of seeds on the screen. Such motion principle determined from the size, shape, and density of seeds and the control parameters: velocity of airflow, oscillation frequency, longitudinal pitch, and transverse pitch of screens. Four fractions of seeds evaluated by kernel weight of seeds outflow from four slots installed at gravity seed cleaner. The kernel weight of each fraction is measured online as the controlled variable of the system.

The control parameters should be adjusted at suitable setpoints before the seed cleaner starts. During the process of separating the seeds, the control parameters are unchangeable. Obviously, such a system is a static, nonlinear and decoupling control system. With the suitable setpoints, the seed cleaner can obtain excellent results. However, the setpoints are very difficult to tune with many control parameters and variable varieties and lots of seeds. Liu et al [4] used the good parameters, which are obtained from experiment with familiar breed and saved in control system, and adjusted the seed cleaner. But it is not flexible for variable breeds and batches of seeds. For finding out the near-optimal values for different breeds and batches of seeds, we propose a new optimal control system.

\section{METHODOLGY FOR OPTIMAL CONTROL}

\subsection{Design of an optimal control system}

One of the important objects for optimal control is how to deal with the search process for near-optimal parameters to control nonlinear system. GAs is the successful option to optimize various control parameters. However, it is very time consuming to obtain fitness values for some control problems as 
it is impractical to run a real system for each combination of control parameters. Due to good generalization abilities, ANNs are often used to model and approximate a real control system. So the ANNs could be used in fitness evaluation rather than the real control system [5]. Fig. 1 shows the block diagram of the hybrid intelligent optimal control system.

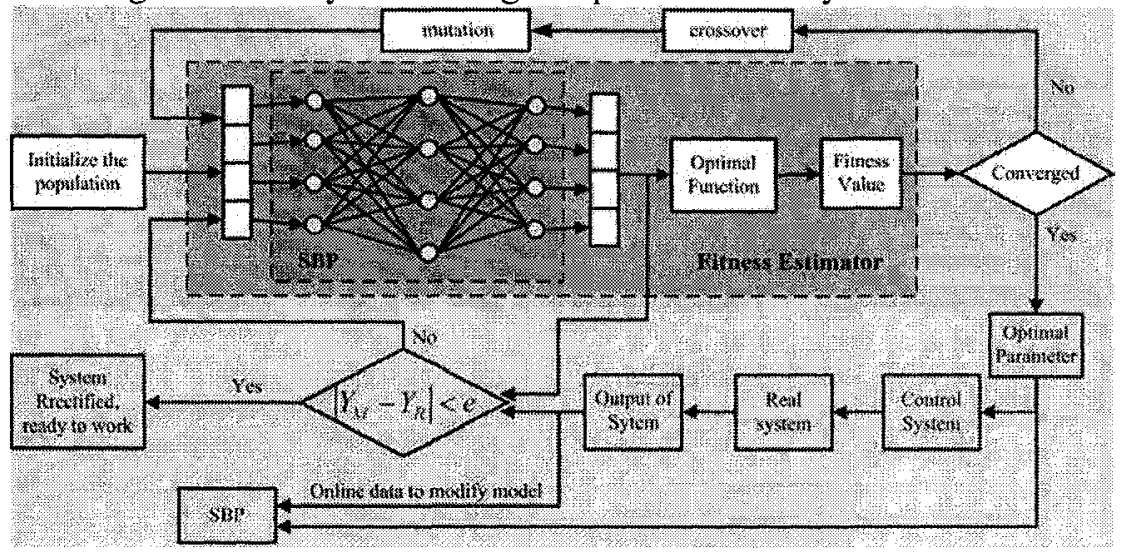

Figure 1. Block diagram of hybrid intelligent optimal control system.

The optimal control is realized through two procedures: identification of seed cleaner system using ANNs and the search for a near-optimal value through simulation of the identified model using GAs.

Because of an optimal value is obtained from a model simulation, the high accuracy of model approximation is badly needed. Therefore, once the approximate error between model and real system exceed the threshold, the ANNs model should be updated with new sample, meanwhile, the search of GAs should be started again. The optimization is accomplished by applying the optimal values to the setpoints in the feedback control system.

\subsection{Sampling based on uniform design}

The training set influences modeling accuracy of ANNs. In theory, an infinite training set would provide an exact measure of test accuracy and discourage overfitting. Hence, it is desirable to incorporate a training set into the learning phase as large as possible. However, training on very large data set is problematic, as training time tends to increase. In particular, sampling large data sets is impractical at this case. A sampling approach based on uniform design has shown to decrease data sets, but reserving the underlying distribution by sampling.

Uniform Design (UD) is an experimental design method proposed by Fang [6]. UD is equivalent to generating a relative small set of design points 
that are uniformly scattered on experiment domain, which reflects selfcontained information of the system. Data sets have more generalization performance. Its main theory is using certain method to design the uniformly scattered point sets instead of Monte Carlo random point sets in problem space.

UD is often expressed as a table, denoted by $\mathrm{U}_{\mathrm{n}}\left(\mathrm{q}^{s}\right)$ : a matrix of $q$ rows and $s$ columns, which means $s$ factors with $q$ levels for each of the factors in the problem space. Generally, $n$ presented as the number of experiment is equal to $q$, namely the number of factor's level. If the level is different among factors, the UD table should be designed as mixed level UD table, for example, table 1 used in our experiment. Its first factor has 12 levels, but the other 3 factors have 6 levels. For detail UD, reference Fang [6].

Table 1. Mixed Level Uniform Design table $\mathrm{U}_{12}\left(12 \times 6^{3}\right)$

\begin{tabular}{lllll}
\hline $\begin{array}{l}\text { No. of } \\
\text { Experiment }\end{array}$ & $\begin{array}{l}\text { Oscillation } \\
\text { Frequency } \\
(450 \sim 1000 \mathrm{~Hz})\end{array}$ & $\begin{array}{l}\text { Longitudinal } \\
\text { Obliquity } \\
\left(1.5^{\circ} \sim 9^{\circ}\right)\end{array}$ & $\begin{array}{l}\text { Screen Airflow } \\
\text { Velocity } \\
(1 \sim 6 \mathrm{~m} / \mathrm{s})\end{array}$ & $\begin{array}{l}\text { Transverse } \\
\text { Obliquity } \\
\left(1^{\circ} \sim 6^{\circ}\right)\end{array}$ \\
\hline 1 & $1(450)$ & $2(3)$ & $6(6)$ & $2(2)$ \\
2 & $2(500)$ & $3(4.5)$ & $5(5)$ & $4(4)$ \\
3 & $3(550)$ & $5(7.5)$ & $4(4)$ & $6(6)$ \\
4 & $4(600)$ & $6(9)$ & $3(3)$ & $2(2)$ \\
5 & $5(650)$ & $1(1.5)$ & $2(2)$ & $4(4)$ \\
6 & $6(700)$ & $3(4.5)$ & $1(1)$ & $6(6)$ \\
7 & $7(750)$ & $4(6)$ & $6(6)$ & $1(1)$ \\
8 & $8(800)$ & $6(9)$ & $5(5)$ & $3(3)$ \\
9 & $9(850)$ & $1(1.5)$ & $4(4)$ & $5(5)$ \\
10 & $10(900)$ & $2(3)$ & $3(3)$ & $1(1)$ \\
11 & $11(950)$ & $4(6)$ & $2(2)$ & $3(3)$ \\
12 & $12(1000)$ & $5(7.5)$ & $1(1)$ & $5(5)$ \\
\hline
\end{tabular}

\subsection{Artificial Neural Network model}

The establishment of the model using SBP (Standard Back-Propagation) is realized through two steps. In the first step, the relative exact approximation of SBP model is identified fast with less sample sets which sampled offline. Next, the approximation of SBP will be trained more accurately using the feedforward modeling approach.

The nonlinear model identified by SBP of seed cleaner system can be expressed as follows:

$$
G=F(X, W)
$$

Where, the input vector is defined $X=[\alpha \beta f v]^{T}$, the output vector is defined $G=\left[\begin{array}{llll}g_{1} & g_{2} & g_{3} & g_{4}\end{array}\right]^{T}$, where $\alpha$ denotes longitudinal obliquity, $\beta$ denotes transverse obliquity, $f$ denotes oscillation frequency and $v$ denotes 
screen airflow velocity. $g_{i}$ represents the kernel weight of fraction $i . \mathbf{W}$ is the weight matrix that is evaluated in the network training process.

Due to the relative less sample sets, the leave-one-out cross validation was used in training process of SBP, and the architecture of SBP was built up by NEUframe from Neuscience, UK.[7]

\subsection{GAs design}

GAs refers to a population-based stochastic search algorithms that are developed from ideas and principles of natural evolution, which is useful for dealing with large complex problems. It does not depend on gradient information and thus are quite suitable for problems where such information is unavailable or very costly to obtain or estimate. Here, the searching process for near-optimal setpoints is using GAs within the nonlinear model identified by ANN. This part just focuses on the fitness value computation.

\subsubsection{Optimal objective function}

There was variability between lots in the percentage of seed contained in each fraction. Generally, the percentages of fractions are given based on the experiment of germination percentage according to the lots, thus, the optimal objective function is determined:

$$
\max f\left(g_{1}, g_{2}, g_{3}, g_{4}\right)=\frac{1}{\left(\left(\lambda_{1} g k-g_{1} k_{1}\right)^{2}+\left(\lambda_{1} g k-g_{1} k_{1}\right)^{2}+\left(\lambda_{1} g k-g_{1} k_{1}\right)^{2}+\left(\lambda_{1} g k-g_{1} k_{1}\right)^{2}\right)}
$$

Where $g_{i}, \lambda_{i}, k_{i}$ is the kernel weight, presumed seeds percentage and grain number of fraction $i, k=k_{1}+k_{2}+k_{3}+k_{4}$.

\subsubsection{Fitness function of GAs}

The fitness value could be computed by combining the formulation (1) and (2). The difference from general GAs is that the variables expressed by chromosome is not directly independent variable of optimal function, but independent variable of optimal function via nonlinear mapping of ANNs.

\section{RESULTS}

The test was done on 5XZW-1.5 type seed cleaner control system with 3 lots of seeds. And the separating result used this optimal approach $(\mathrm{O})$ was compared with that of mechanical adjustment (M). 
Table 2. The comparison of testing results for various approaches

\begin{tabular}{|c|c|c|c|c|c|c|c|c|c|c|c|c|}
\hline \multirow[t]{2}{*}{$\begin{array}{l}\text { Seed } \\
\text { lot }\end{array}$} & \multicolumn{2}{|c|}{$\begin{array}{l}\text { Fraction } \\
1(\%)\end{array}$} & \multicolumn{2}{|c|}{$\begin{array}{l}\text { Fraction } \\
2(\%)\end{array}$} & \multicolumn{2}{|c|}{$\begin{array}{l}\text { Fraction } \\
3(\%)\end{array}$} & \multicolumn{2}{|c|}{$\begin{array}{l}\text { Fraction } \\
4(\%)\end{array}$} & \multicolumn{2}{|c|}{$\begin{array}{l}\operatorname{Max} f(g 1, g 2, g 3, \\
g 4)\end{array}$} & \multicolumn{2}{|c|}{$\begin{array}{l}\text { Percent } \\
\text { germination }\end{array}$} \\
\hline & $\mathrm{M}$ & $\mathrm{O}$ & $\mathrm{M}$ & $\mathrm{O}$ & $\mathbf{M}$ & $\mathrm{O}$ & $\mathrm{M}$ & $\mathrm{O}$ & $\mathrm{M}$ & $\mathrm{O}$ & $\mathrm{M}$ & 0 \\
\hline $\mathrm{A}$ & 40 & 48 & 27 & 30 & 21 & 15 & 12 & 7 & 91.5 & 95.7 & 91.3 & 952 \\
\hline B & 35 & 42 & 29 & 27 & 17 & 18 & 19 & 13 & 93 & 972 & 90.8 & 94.8 \\
\hline $\mathrm{C}$ & 42 & 49 & 24 & 25 & 22 & 15 & 12 & 11 & 90.5 & 96.8 & 92.9 & 97.3 \\
\hline
\end{tabular}

Quality of seed fractions was defined finally by determining the percent germination of first two fractions. The percent germination in table 2 shows that the optimal control approach is consistently higher than the adjustment with control parameters saved before.

\section{CONCLUSION}

In this paper, a hybrid intelligent technique based on ANNs and GAs was applied to the optimal control. First, seed cleaner system was identified using SBP. For minimizing approximate error, uniform design plans sampling the sample sets of SBP, and leave-one-out cross validation was used in training process of SBP. Such a trained network becomes a specialised process simulation tool which is valuable in prediction and optimization of control system. Prediction of process output can be used to determine whether some separated result of seeds will be satisfactory, given the input conditions and process parameters. Next, near-optimal setpoints of the seed cleaner, which maximize the objective function, were sought from among the numerous values of the objective function obtained from the simulation. Thus, this hybrid intelligent approach is suitable for the optimal control of complex systems, e.g. seed cleaner system.

\section{REFERENCES}

1. Wang, K., (2005), Applied Computational Intelligence to Intelligence Manufacturing Systems, Advanced Knowledge International (ISBN 0-9751004-9-1).

2. Tarik C., Mehmet B. Y., and Mehmet B. (2005), A neuro-genetic approach to design and planning of a manufacturing cell, $J$ of Intelligent Manufacturing, vol. 16, pp. 255-274.

3. Holland, J. H., (1989), Searching nonlinear functions for high values, Applied Mathematics and Computation, vol. 32, pp. 255-274.

4. Liu, C. L. et al., (1997), Study on the design and adaptive control system of the gravity seed cleaner, Trans. the Chinese Society of Agricultural Machinery, v28,no. 1, p74-77.

5. Yao, X., (1999), Evolving artificial neural networks, Proceedings of the IEEE, 87(9):14231447.

6. Fang, K. T. and Wang, Y., (1994), Number-theoretic Methods in Statistics. Chapman \& Hall.

7. NEUframe User's Guide, www.neusciences.com 